\title{
COGNITIVE
} SCIENCE

\section{Area activation: a computational model of saccadic selectivity in visual search}

\author{
Marc Pomplun $^{\mathrm{a}, *}$, Eyal M. Reingold ${ }^{\mathrm{b}, 1}$, Jiye Shen ${ }^{\mathrm{b}, 1}$ \\ ${ }^{a}$ Department of Computer Science, University of Massachusetts, 100 Morrissey Boulevard, \\ Boston, MA 02125-3393, USA \\ ${ }^{\mathrm{b}}$ Department of Psychology, University of Toronto, 100 St. George Street, Toronto, Ont., Canada M5S 3G3
}

Received 13 December 2001; received in revised form 4 September 2002; accepted 17 January 2003

\begin{abstract}
The Area Activation Model (Pomplun, Reingold, Shen, \& Williams, 2000) is a computational model predicting the statistical distribution of saccadic endpoints in visual search tasks. Its basic assumption is that saccades in visual search tend to foveate display areas that provide a maximum amount of taskrelevant information for processing during the subsequent fixation. In the present study, a counterintuitive prediction by the model is empirically tested, namely that saccadic selectivity towards stimulus features depends on the spatial arrangement of search items. We find good correspondence between simulated and empirically observed selectivity patterns, providing strong support for the Area Activation Model. (C) 2003 Cognitive Science Society, Inc. All rights reserved.
\end{abstract}

Keywords: Visual search; Eye movements; Saccadic selectivity; Computational modeling; Simulation

\section{Introduction}

In a visual search task, participants are presented with a search display showing a set of search items and are typically asked to decide whether the display includes a designated target item. There is growing literature of eye-movement studies in visual search demonstrating saccadic selectivity, that is, a bias of saccadic endpoints towards a particular type of distractor (e.g., Findlay, 1997; Hooge \& Erkelens, 1999; Luria \& Strauss, 1975; Motter \& Belky, 1998; Pomplun, Reingold, \& Shen, 2001b; Scialfa \& Joffe, 1998; Shen, Reingold, \& Pomplun, 2000;

\footnotetext{
${ }^{*}$ Corresponding author. Tel.: +1-617-287-6443; fax: +1-617-287-6433.

E-mail addresses: marc@cs.umb.edu (M. Pomplun), reingold@psych.utoronto.ca (E.M. Reingold), jiye@psych.utoronto.ca (J. Shen).

${ }^{1}$ Tel.: +1-416-978-3990; fax: +1-905-822-3336.
} 
Williams, 1967; Williams \& Reingold, 2001, but see Zelinsky, 1996). For example, if the target is defined by a certain combination of color and shape, the distractors sharing color with the target may receive substantially more fixations than those sharing shape with the target (e.g., Williams \& Reingold, 2001).

In all of these studies, saccadic selectivity - the proportion of saccades directed to each distractor type-was calculated by assigning saccadic endpoints to the closest display item. One problem with this method is that it ignores the possibility that the placement of saccadic endpoints is guided by the combined potential relevance of several nearby display items. However, such an influence of distractor grouping on saccadic selectivity is likely given the well-documented influence of distractor grouping on visual search efficiency (e.g., Beck, 1993; Duncan \& Humphreys, 1989; Grossberg, Mingolla, \& Ross, 1994; Humphreys \& Müller, 1994; Poisson \& Wilkinson, 1992; Treisman, 1982). For example, Treisman (1982) demonstrated that conjunction search was much easier when the distractor items were grouped by kind, indicating that subjects may perform a serial search across groups, and that search within distinct groups may be conducted in parallel (see also Grossberg et al., 1994). Similarly, the attentional-engagement theory by Duncan and Humphreys $(1989,1992)$ suggested that visual search is facilitated by efficient distractor grouping but is hindered by grouping between the target and distractors.

Thus, in the present study we set out to study the influence of distractor grouping on saccadic selectivity. Our investigation was conducted within the framework of a computational approach toward simulating saccadic selectivity in visual search, which we refer to as the Area Activation Model (see Pomplun, Reingold, Shen, \& Williams, 2000). Accordingly, in the remainder of the introduction we briefly describe the concepts underlying the Area Activation Model and the way in which it was implemented in the present study.

A key concept underlying the Area Activation Model is related to the notion that, during a fixation, the distribution of visual processing resources - the "fixation field" - is a two-dimensional Gaussian function centered at the fixation point (cf. Pomplun, Ritter, \& Velichkovsky, 1996). As demonstrated by Pomplun, Reingold, and Shen (2001a) the size of the fixation field is likely to decrease with an increase in task difficulty and attentional or foveal load. In addition, fixation field may increase with practice as participants gain expertise and familiarity with the stimulus domain (Charness, Reingold, Pomplun, \& Stampe, 2001; Reingold, Charness, Pomplun, \& Stampe, 2001; Reingold, Charness, Schultetus, \& Stampe, 2001; Scialfa \& Joffe, 1998). Furthermore, item density and heterogeneity are likely to have an influence on the size of the fixation field with widely dispersed, homogeneous items producing larger fixation fields than densely packed, heterogeneous items.

The basic assumption underlying the Area Activation Model is that saccades in visual search tend to be directed toward display areas that provide a maximum amount of task-relevant information. Based on the assumed spatial distribution of processing resources during fixations and the relevance of different items to the search task it is possible to compute the informativeness and therefore the activation value of every point in the display, with more highly activated positions being more likely to be fixated than less activated positions. Activation peaks correspond to positions that, if fixated, would optimize the acquisition of task-relevant information. Thus, in order to predict the statistical distribution of saccadic endpoints, the Area Activation Model employs an area-based activation map, rather than an item-based activation map. 
The concept of an area-based activation map adopted by the Area Activation Model is partially inspired by empirical evidence derived from behavioral and neurophysiological studies of the saccadic system. Specifically, several studies reported that when two potential saccadic targets are presented in relatively close proximity, rather than fixating one of these targets, the first saccade endpoints tend to be directed toward intermediate locations. This effect, referred to as the center of gravity or global effect, appears to be very general and was demonstrated with both voluntary (Coren \& Hoenig, 1972) and reflexive saccades (e.g., Deubel, Wolf, \& Hauske, 1984; Findlay, 1981, 1982; Findlay, Brogan, \& Wenban-Smith, 1993; Ottes, van Gisbergen, \& Eggermont, 1984). Most important for the present context, global effects were also observed in visual search when targets were presented in neighboring locations (Findlay, 1997; Findlay \& Gilchrist, 1997; Zelinsky, Rao, Hayhoe, \& Ballard, 1997). Among the current models of saccadic control in overt visual search (e.g., Findlay \& Walker, 1999; Itti \& Koch, 2000; Rao, Zelinsky, Hayhoe, \& Ballard, 2002), both the Findlay and Walker model and the Rao et al. model address the global effect and related phenomena. Both models postulate a spatially-coded salience map, which directs saccades to the center of the most salient areas (i.e., groups of items) instead of the most salient single items. Thus, the mechanism of spatial integration of visual input proposed by the Area Activation Model is consistent with currently available evidence and theoretical frameworks concerning saccade generation in simple and complex visual tasks.

In the following paragraphs we provide a step-by-step overview of how to apply the Area Activation Model to a given visual search task in order to simulate saccade amplitude, the distribution of saccadic endpoints, and saccadic selectivity. A precise mathematical description of the model and its implementation is provided in Appendix A, and its application to another visual search task is described in Pomplun et al. (2000).

\subsection{Step 1: estimation of guidance by distractor types}

In order to compute the informativeness of any potential fixation position in the search display, we need to know which type of distractor guides the visual search process. This can be determined in a preliminary study using the distractor and target types in question, including a dissimilar distractor type that does not share any features with the target. Any distractor type that receives significantly more fixations than the dissimilar type is assumed to guide the search process. In many search tasks - as in the task employed in the present study-there is guidance by only one distractor type, so that the model can disregard the other distractor types, and no further parameters have to be estimated. In the case of multiple distractor types showing distinct strengths of guidance, the relative contribution of each distractor dimension has to be estimated and incorporated into the model as an extra parameter. Pomplun et al. (2000) applied this procedure to a triple-conjunction search task and showed how the model can predict the amount of simultaneous guidance by multiple dimensions from data obtained in single-dimension guidance conditions.

\subsection{Step 2: estimation of the fixation field size}

A crucial variable of the Area Activation Model, fixation field size, has to be estimated separately for each experimental condition. To achieve this, we exploit the functional 
relationship between fixation field size and the number of fixations per trial generated by the model: A larger fixation field always leads to fewer fixations per trial, because in the activation map larger groups of guiding items merge into a single peak. The computation is performed by an iterative algorithm that receives as its input the number of fixations obtained in the corresponding trials in a preliminary study. The algorithm starts with an arbitrary estimate of fixation field size. Based on this size, for each trial the algorithm computes an activation map and counts its peaks to determine the number of fixations that the model generates. If the generated average number of fixations is considerably smaller or greater than the empirically obtained one, the assumed fixation field size is decreased or increased, respectively, and the next iteration begins. The step size for fixation field adjustment is halved after each iteration, and the algorithm terminates as soon as the difference between empirical and simulated number of fixations falls below a certain threshold. As we have shown in earlier work (Pomplun et al., 2000), it is possible to determine the fixation field size in a preliminary study and then predict saccadic selectivity and saccade amplitude for different subjects performing a similar task. In this case, the model does not require any empirically obtained parameters from the subjects in order to predict their saccadic behavior.

\subsection{Step 3: simulation of scan paths}

For each search display in the experiment, an algorithm computes the activation map and multiple scan paths. Since the calculation of fixation positions is carried out as a statistical process, it is advantageous to generate a large number of scan paths per display in order to obtain reliable data. To compute a visual scan path, we not only need to determine the fixation positions, but also the order in which they are visited. As indicated by empirical studies (e.g., Zelinsky, 1996), the choice of such an order is governed by principles of spatial optimization. The following simple rule in the current implementation of the Area Activation Model reflects such a principle: The next fixation target is always the activation peak closest to the current gaze position that has not been visited yet. This method of local minimization of scan path length ("Greedy Heuristic") has been shown to be useful in modeling empirical scanning sequences (e.g., Pomplun, 1998).

\subsection{Overview of the current study}

In the present paper we further evaluate the Area Activation Model by empirically testing one of its strongest predictions. Specifically, if saccadic selectivity is measured in the typical manner (i.e., by assigning saccadic endpoints to the closest display item) then grouping of guiding search items should increase saccadic selectivity toward them. This is because in the absence of grouping, the peaks in the area-based activation map do not necessarily coincide with the positions of the guiding distractors, but may have a distractor of another type as their nearest neighbor (see Fig. 1b). Therefore, the measured saccadic selectivity for guiding items is relatively low. However, grouping guiding distractors leads to a higher probability that peaks would correspond to the position of a guiding distractor producing higher saccadic selectivity towards them (see Fig. 1a). 
(a)
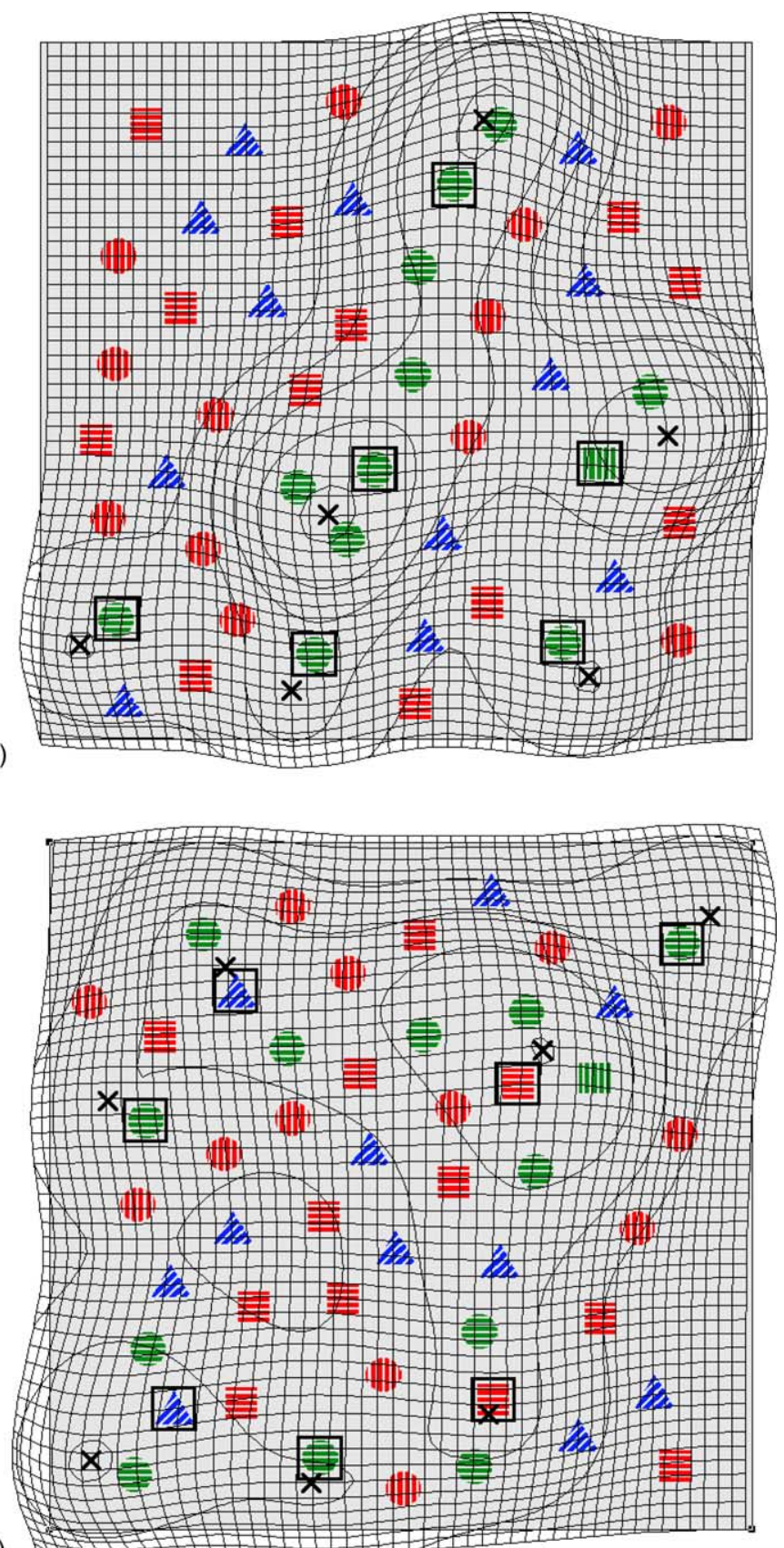

(b)

Fig. 1. Sample displays created to induce high color guidance (panel a) or low color guidance (panel b), with the area activation "landscape" superimposed on them. In either display, the target is a green, vertically striped square, and the color green is assumed to exclusively guide visual search. The peaks in the activation map are marked with black crosses. Squares indicate the closest item to the projection of each peak onto the stimulus plane. While in the high guidance display (panel a) all six peaks are closest to a guiding green item, in the low guidance display (panel b) this is the case for only three out of seven peaks. 
In order to test this hypothesis, we employed a triple-conjunction search task that was introduced by Pomplun et al. (2001b) and created two groups of visual search displays. Although all of these displays contained the same set of search items, they were-according to the Area Activation Model-supposed to induce relatively strong saccadic selectivity ("high guidance displays") or relatively weak saccadic selectivity ("low guidance displays") towards the guiding distractor type. By using an independent clustering measure (see Appendix B) we verified that high and low guidance displays differed significantly in terms of clustering and that the model's prediction of saccadic selectivity was strongly correlated with clustering values (see method section for details). We then proceeded to empirically contrast saccadic selectivity across high and low guidance displays and documented a significant selectivity difference in the predicted direction between these two groups of displays consistent with the Area Activation Model.

\section{Method}

\subsection{Participants}

Eight undergraduate students from the University of Toronto were tested individually in 1-h sessions. All participants had normal or corrected-to-normal vision. They were naïve with respect to the purpose of the study and were paid $\$ 10 \mathrm{Cdn}$. for their participation.

\subsection{Apparatus}

Eye movements were recorded with the SR Research Ltd. EyeLink system, which operates at a sampling rate of $250 \mathrm{~Hz}$ (4 ms temporal resolution) and measures a participant's gaze position with an average error of less than $0.5^{\circ}$ of visual angle. Stimuli were presented on a $19 \mathrm{in}$. Samsung SyncMaster 900P monitor with a refresh rate of $120 \mathrm{~Hz}$ and a screen resolution of $800 \times 600$ pixels.

\subsection{Materials}

The stimulus displays were identical to some of those used in Pomplun et al. (2001b). They consisted of geometrical objects with features varying along three dimensions-color (red vs. green), shape (circle vs. square), and orientation (vertical vs. horizontal gratings). For each participant, one of these eight item types served as search target. The identity of the target was counterbalanced across participants. The target and the distractors shared one feature (color, shape, or orientation). The fourth distractor type — a blue, diagonally striped triangleshared no feature with the target and was originally used to derive base rates for the saccadic selectivity analysis. Target-absent displays consisted of 12 instances of each distractor type (three similar and one dissimilar type), for a total of 48 items per display. In target-present trials, a target-absent display was first generated and then one of the distractors was randomly chosen to be replaced with the target item. All stimuli were presented in a $15.5^{\circ} \times 15.5^{\circ}$ field at a viewing distance of $80 \mathrm{~cm}$. The target was never presented within the central $2.7^{\circ} \times$ $2.7^{\circ}$ field of the display. Each item was about $1^{\circ}$ in diameter, and the minimum distance 
between the centers of neighboring items was $1.8^{\circ}$. From the study by Pomplun et al. (2001b) we knew the empirically measured number of fixations per trial in these displays and that the only dimension guiding search was color. This knowledge made it possible to have a computer program generate random search displays, simulate saccades according to the Area Activation Model, and calculate saccadic selectivity. Displays in the top 5\% of color selectivity were assigned to the "high guidance stimuli" group (see Fig. 1a), and displays in the bottom $5 \%$ were assigned to the "low guidance stimuli" group (see Fig. 1b). High guidance stimuli generally showed strongly clustered color distractors, whereas in low guidance stimuli the color distractors were widely dispersed. To quantify this effect, we defined a measure $\rho$ indicating the strength of color-distractor clustering (see Appendix B). The clustering of color distractors was significantly stronger in the high guidance stimuli $(\rho=1.24)$ than in the low guidance stimuli $(\rho=0.93), t(478)=48.23, p<.001$. Furthermore, in 1,000 randomly generated displays we found a remarkable correlation between the clustering of color distractors and the model's simulation of saccadic color selectivity $(r=.91, p<.001)$. The design of the experiment included four cells that consisted of the combinations of target status (present vs. absent) and stimulus type (high vs. low guidance). Following 16 practice trials, each participant performed 120 trials for each cell administered in eight blocks of 60 trials. The order of stimulus displays was completely randomized except that there were no more than four successive displays of a given target status.

\subsection{Procedure}

Prior to the start of the experiment, participants were informed about the identities of the target and distractor items and about their task - to search for the target item and indicate whether it was present or absent by pressing an appropriate button as quickly and as accurately as possible. A calibration procedure was performed at the beginning of the experiment, and each trial started with a gaze position drift correction (see Stampe, 1993). Participants were instructed to fixate on a dot shown in the center of the screen and then press a button to start the next trial. The trial terminated if participants pressed one of the response buttons or if no response was made within $30 \mathrm{~s}$. The particular buttons used to indicate target presence or absence were counterbalanced across participants.

\section{Results}

Only target-absent trials were included in the analysis, because the results from target-absent trials can be interpreted more clearly than those from target-present trials, where the presence of the target may influence search behavior (see Zelinsky, 1996). There were $2.0 \%$ incorrect responses in the high guidance condition and $1.1 \%$ in the low guidance condition, with no significant difference between the two conditions, $t<1$. These trials were excluded from further analysis, as well as trials with a saccade or a blink overlapping the onset of a display ( $1.4 \%$ for high and $1.2 \%$ for low guidance, $t<1$ ) or response times (RTs) that were more than three standard deviations above or below the mean $(0.8 \%$ in each condition, $t<1)$. Since the model does not currently address refixations of the same items, we only analyzed the first five 
fixations in each trial, which include only a small proportion of refixations $(0.6 \%$ for high and $0.7 \%$ for low guidance).

In the high guidance condition, there were shorter RTs $(1,540 \mathrm{~ms})$ and fewer fixations per trial (5.73) than in the low guidance condition $(1,775 \mathrm{~ms}$ and 6.94 fixations), $t(7)=8.64$, $p<.001$ and $t(7)=9.92, p<.001$, respectively, indicating higher search efficiency in the high guidance condition. This result can be explained by differences in activation maps between the two conditions (cf. Fig. 1): while high guidance stimuli contained clustered color distractors inducing focused activation maps, low guidance stimuli induced spread-out activation requiring more fixations to process the whole display. This is in line with the finding of shorter saccades in the high guidance condition $\left(4.94^{\circ}\right)$ than in the low guidance condition $\left(6.27^{\circ}\right), t(7)=7.09$, $p<.001$. Fig. 2a presents the distribution of empirically obtained saccade amplitude in each condition.

The major question of the present study was whether, as predicted by the Area Activation Model, participants' saccadic selectivity towards the color dimension was higher in the high guidance condition than in the low guidance condition. To investigate this, the selectivity of participants' eye movements was determined by the standard algorithm for selectivity studies (e.g., Zelinsky, 1996). For every trial, we calculated the distance between each saccadic endpoint and every item in the display. Each saccadic endpoint was assigned to the item nearest to it, so that for each participant, the proportion of saccades directed at each distractor type-the saccadic selectivity - could be computed.

Most importantly, as predicted by the Area Activation Model, selectivity towards color was significantly higher in the high guidance condition than in the low guidance condition, $t(7)=7.65, p<.001$. The analysis of saccadic selectivity in the high guidance condition (color distractors: $60.2 \%$; shape: $16.0 \%$; orientation: $10.8 \%$; dissimilar: $13.0 \%$ ) and the low guidance condition (color: $45.1 \%$; shape: $21.3 \%$; orientation: $16.7 \%$; dissimilar: $16.9 \%$ ) revealed significant guidance (high guidance condition: $F(3,21)=68.34, p<.001$; low guidance condition: $F(3,21)=26.63, p<.001)$. In either condition, color was the only dimension to guide visual search, replicating the findings by Pomplun et al. (2001b). This was indicated by selectivity being higher towards color than to any other dimension (high guidance condition: all $t(7)>6.77, p<.01$; low guidance condition: all $t(7)>3.94$, $p<.05$ ) and no significant differences in selectivity across the other dimensions (high guidance condition: all $t(7)<2.88, p>.1$; low guidance condition: all $t(7)<1.75$, $p>.5)$.

Fig. 2c shows color selectivity of saccadic endpoints as a function of saccade amplitude in the high and low guidance conditions. As can clearly be seen, the difference in color selectivity between the two conditions decreases with increasing saccade amplitude. This is due to a general decrease in selectivity with longer saccades.

We used the empirical results obtained in the present experiment to further evaluate the Area Activation Model. The iterative algorithm determined fixation field sizes of $1.43^{\circ}$ in the high guidance condition and $1.72^{\circ}$ in the low guidance condition to match the empirically obtained number of fixations per trial in each condition. The model was then "presented" with exactly the same displays that were used in the present experiment and generated 1,000 visual scan paths for each of them to derive reliable saccade amplitude distributions and mean saccadic selectivity values. 

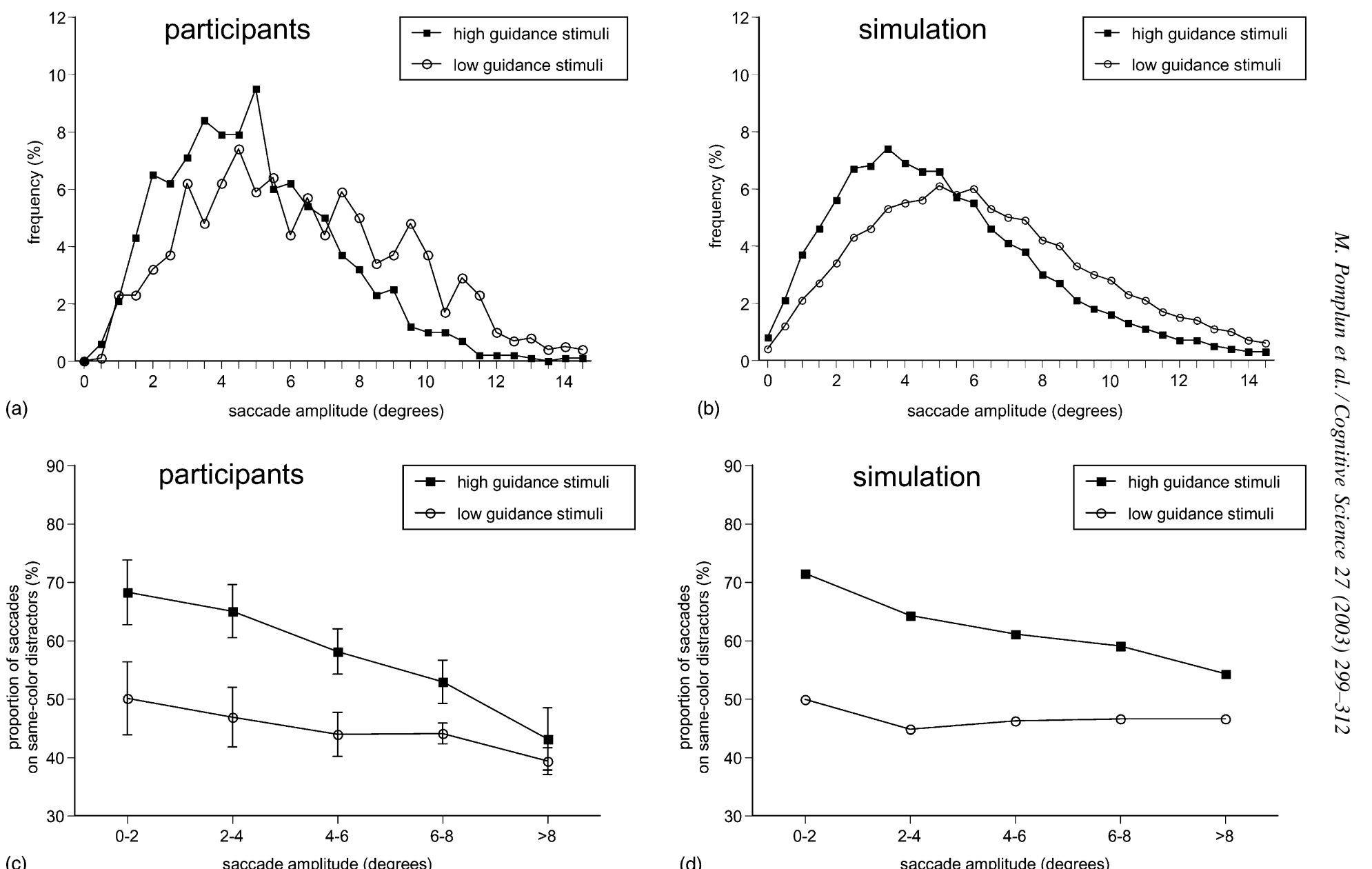

(c)

saccade amplitude (degrees)

(d)

Fig. 2. Distribution of saccade amplitude (panels a and b) and saccadic selectivity towards color (panels c and d) in the high and low guidance conditions for empirical (panels a and c) and simulated saccades (panels b and d). 
Fig. 2b shows saccade amplitude histograms as simulated by the model for the high and low guidance conditions. Although due to a larger number of samples these histograms are smoother than the empirical ones displayed in Fig. 2a, it is obvious that the model's simulation of the two distributions is quite accurate (high guidance: $r^{2}=.95$, RMSD $=0.89 \%$; low guidance: $\left.r^{2}=.86, \mathrm{RMSD}=0.83 \%\right)$. Moreover, there is a good fit in the simulation of mean saccade amplitude in both the high guidance condition (empirical: $4.94^{\circ}$; simulated: $5.33^{\circ}$ ) and the low guidance condition (empirical: $6.27^{\circ}$; simulated: $6.49^{\circ}$ ). The model's simulation of color selectivity as a function of saccade amplitude is shown in Fig. 2d. By comparing it to the corresponding empirical data in Fig. 2c, it can be seen that the overall patterns match quite well. All in all, the results of the simulation are clearly compatible with the empirical data.

\section{Discussion}

The present results are consistent with the well-documented influence of distractor grouping on visual search efficiency (e.g., Beck, 1993; Duncan \& Humphreys, 1989; Grossberg et al., 1994; Humphreys \& Müller, 1994; Poisson \& Wilkinson, 1992; Treisman, 1982). The current findings suggest that this link between distractor grouping and search efficiency is also reflected in the pattern of saccadic selectivity. Specifically, we demonstrated that grouping the guiding search items in a visual search task could substantially increase saccadic selectivity for them. This pattern of results provides support for the Area Activation Model and the concept of smooth area-based activation maps rather than item-based activation maps. Moreover, as shown by a computer simulation, the Area Activation Model is able to generate both saccade amplitude distributions and saccadic selectivity patterns that closely match the empirical ones.

However, it should be clearly stated that the current implementation of the Area Activation Model is not intended to provide a universal model of visual search. Rather the model in its current state is not very complex and focuses on some particular, previously unexplored issues such as the mapping of fixations to items. Other important issues have not been addressed yet. For instance, the model does not incorporate any assumptions concerning the role of memory in visual search (see Findlay, Brown, \& Gilchrist, 2001; Frens, van der Geest, \& Hooge, 2000; Gilchrist, North, \& Hood, 2001; Horowitz \& Wolfe, 1998, 2001, 2003; Peterson, Kramer, Wang, Irwin, \& McCarley, 2000) and consequently is unable to deal with refixations on the same items. Accordingly, we had to restrict our evaluation of the model to the first five fixations in each trial, which rarely involve refixations. We also know that the present model does not perform well for rapid search processes that occur, for example, in displays with very few highly salient guiding items that are far apart from each other. In such cases, the Area Activation Model assumes extremely large fixation fields so that many simulated saccades land between guiding items. However, empirical data show that under these conditions most saccades are directed at the guiding items rather than at the areas between them (Shen et al., 2000). Evidently, there are neurophysiological and psychophysical limits to the size of the fixation field that need to be incorporated into the model.

Although more research is required to further evaluate and develop the Area Activation Model, the finding of grouping effects on saccadic selectivity constitutes an important empirical benchmark for any present or future theory of visual search. This result is clearly inconsistent 
with any account of saccadic selectivity that is restricted to purely item-based activation maps. Thus, the Area Activation Model can be considered a step towards an adequate, universal model of visual search. In particular, we believe that currently dominant visual search models, such as the Guided Search Theory (e.g., Cave \& Wolfe, 1990; Wolfe, 1994, 1996; Wolfe, Cave, \& Franzel, 1989; Wolfe \& Gancarz, 1996) should be extended to explain grouping effects, possibly by means of an area-based activation map.

\section{Acknowledgments}

Preparation of this paper was supported by a grant to Eyal M. Reingold from the Natural Science and Engineering Research Council of Canada (NSERC) and a grant to Marc Pomplun from the Deutsche Forschungsgemeinschaft (DFG).

\section{Appendix A. Quantitative description of the model}

To provide a mathematical description of the Area Activation Model, let us define a search display to comprise $N$ guiding items with positions $\left(x_{n}, y_{n}\right), n \in\{1, \ldots, N\}$. This definition is only applicable to displays with exactly one homogeneous group of distractors guiding visual search, such as the color distractors in the present study (see Pomplun et al., 2000, for a definition covering more complex guidance patterns). The fixation field is defined as a two-dimensional Gaussian function with standard deviation $\sigma_{f}$ centered at the fixation point.

The activation map is given by a function $m(x, y)$, which yields the amount of information that could be processed while fixating at position $(x, y)$ in the display. This amount is calculated as the sum of visual processing resources applied to each guiding item in the display, based on a Gaussian distribution of resources for processing with its center at position $(x, y)$ and standard deviation $\sigma_{f}$ :

$$
m(x, y)=\sum_{n=1}^{N} \exp \left[-\frac{\left(x-x_{n}\right)^{2}+\left(y-y_{n}\right)^{2}}{2 \sigma_{f}^{2}}\right]
$$

Each local maximum (peak) of $m(x, y)$ is assumed to attract exactly one fixation. These peaks are located at the positions $\left(x_{p}, y_{p}\right)$ meeting the following requirement:

$$
\exists \varepsilon>0:\left|x-x_{p}\right|+\left|y-y_{p}\right|<\varepsilon \Rightarrow m\left(x_{p}, y_{p}\right)>m(x, y) \forall x, y
$$

For most search displays, this set of fixation positions $\left(x_{p}, y_{p}\right)$ optimizes the acquisition of information. The visual system, however, is not always able to generate accurate activation maps and to program saccades that exactly hit these optimal targets. It is therefore adequate to model the choice of fixation positions as a statistical process: The probability of a fixation to occur at position $(x, y)$ decreases with increasing distance between $(x, y)$ and the nearest peak in the activation map. It is reasonable to assume that this decrease is steeper if the fixation field is smaller, that is, the search process is more focused. The Area Activation Model accounts for this fact in the most straightforward way: Decrease in fixation probability with increasing 
distance from an activation peak is modeled by the same Gaussian function that describes the decrease in visual processing resources with increasing distance from fixation.

The calculation of actual fixation points also includes simulating the measurement error of the eye-tracker system, which is assumed to be normally distributed with a constant standard deviation $\sigma_{m}$. In the present study, $\sigma_{m}$ was set to $0.5^{\circ}$, reflecting the typical precision of the EyeLink system used in the experiments. Taken together, the probability distribution $p(x, y)$ of actual fixation points for an activation peak $\left(x_{p}, y_{p}\right)$ is given by the following formula:

$$
p(x, y)=\frac{1}{2 \pi\left(\sigma_{f}^{2}+\sigma_{m}^{2}\right)} \exp \left[-\frac{\left(x-x_{p}\right)^{2}+\left(y-y_{p}\right)^{2}}{2\left(\sigma_{f}^{2}+\sigma_{m}^{2}\right)}\right]
$$

\section{Appendix B. The clustering measure}

Let a search display comprise $N$ guiding items with positions $\left(x_{n}, y_{n}\right), n \in\{1, \ldots, N\}$ and $M$ non-guiding items with positions $\left(u_{m}, v_{m}\right), m \in\{1, \ldots, M\}$, where $M, N \geq 2$. Then our measure of clustering $\rho$ for the set of guiding items is given by the following formula:

$$
\rho=\frac{N-1}{2 M} \frac{\sum_{n=1}^{N} \sum_{m=1}^{M} \sqrt{\left(x_{n}-u_{m}\right)^{2}+\left(y_{n}-v_{m}\right)^{2}}}{\sum_{n=2}^{N} \sum_{\eta=1}^{n-1} \sqrt{\left(x_{n}-x_{\eta}\right)^{2}+\left(y_{n}-y_{\eta}\right)^{2}}}
$$

The variable $\rho$ is the quotient of the average Euclidean distance between a guiding and a non-guiding item and the average distance between any two guiding items. A greater value of $\rho$ indicates longer distances between guiding and non-guiding items as compared to the distances within the group of guiding items. This, in turn, signifies that in the search display the guiding items are grouped more tightly.

\section{References}

Beck, J. (1993). The British aerospace lecture: Visual processing in texture segregation. In D. Brogan, A. Gale, et al. (Eds.), Visual search 2 (pp. 1-35). Philadelphia: Taylor \& Francis.

Cave, K. R., \& Wolfe, J. M. (1990). Modeling the role of parallel processing in visual search. Cognitive Psychology, $22,225-271$.

Charness, N., Reingold, E. M., Pomplun, M., \& Stampe, D. M. (2001). The perceptual aspect of skilled performance in chess: Evidence from eye movements. Memory \& Cognition, 29, 1146-1152.

Coren, S., \& Hoenig, P. (1972). Effect of non-target stimuli on the length of voluntary saccades. Perceptual and Motor Skills, 34, 499-508.

Deubel, H., Wolf, W., \& Hauske, G. (1984). The evaluation of the oculomotor error signal. In A. G. Gale \& F. Johnson (Eds.), Theoretical and applied aspects of eye movement research (pp. 55-62). Amsterdam: Elsevier.

Duncan, J., \& Humphreys, G. W. (1989). Visual search and stimulus similarity. Psychological Review, 96, 433-458.

Duncan, J., \& Humphreys, G. W. (1992). Beyond the search surface: Visual search and attentional engagement. Journal of Experimental Psychology: Human Perception \& Performance, 18, 578-588.

Findlay, J. M. (1981). Local and global influences on saccadic eye movements. In D. F. Fisher, R. A. Monty, \& J. W. Senders (Eds.), Eye movements, cognition and visual perception. Mahwah, NJ: Erlbaum.

Findlay, J. M. (1982). Global processing for saccadic eye movements. Vision Research, 22, 1033-1045.

Findlay, J. M. (1997). Saccade target selection during visual search. Vision Research, 37, 617-631. 
Findlay, J. M., Brogan, D., \& Wenban-Smith, M. (1993). The visual signal for saccadic eye movements emphasizes visual boundaries. Perception \& Psychophysics, 53, 633-641.

Findlay, J. M., Brown, V., \& Gilchrist, I. D. (2001). Saccade target selection in visual search: The effect of information from the previous fixation. Vision Research, 41, 87-95.

Findlay, J. M., \& Gilchrist, I. D. (1997). Spatial scale and saccade programming. Perception, 26, 1159-1167.

Findlay, J. M., \& Walker, R. (1999). A model of saccade generation based on parallel processing and competitive inhibition. Behavioral and Brain Science, 22, 661-721.

Frens, M., van der Geest, J., \& Hooge, I. T. C. (2000). Inhibition of saccade return. Perception. CVP abstract, S1.

Gilchrist, I. D., North, A., \& Hood, B. (2001). Is visual search really like foraging? Perception, 30, 1459-1464.

Grossberg, S., Mingolla, E., \& Ross, W. (1994). A neural theory of attentive visual search: Interactions of boundary, surface, spatial, and object representations. Psychological Review, 101, 470-489.

Hooge, I. T., \& Erkelens, C. J. (1999). Peripheral vision and oculomotor control during visual search. Vision Research, 39, 1567-1575.

Horowitz, T. S., \& Wolfe, J. M. (1998). Visual search has no memory. Nature, 394, 575-577.

Horowitz, T. S., \& Wolfe, J. M. (2001). Search for multiple targets: Remember the targets, forget the search. Perception \& Psychophysics, 63, 272-285.

Horowitz, T. S., \& Wolfe, J. M. (2003). Memory for rejected distractors in visual search? Visual Cognition (in press).

Humphreys, G. W., \& Müller, H. J. (1994). Search via recursive rejection (SERR): A connectionist model of visual search. Cognitive Psychology, 25, 43-110.

Itti, L., \& Koch, C. (2000). A saliency-based search mechanism for overt and covert shifts of visual attention. Vision Research, 40, 1489-1506.

Luria, S. M., \& Strauss, M. S. (1975). Eye movements during search for coded and uncoded targets. Perception \& Psychophysics, 17, 303-308.

Motter, B. C., \& Belky, E. J. (1998). The guidance of eye movements during active visual search. Vision Research, $38,1805-1815$.

Ottes, F. P., van Gisbergen, J. A. M., \& Eggermont, J. J. (1984). Metrics of saccadic responses to double stimuli: Two different modes. Vision Research, 24, 1169-1179.

Peterson, M. S., Kramer, A. F., Wang, R. F., Irwin, D. E., \& McCarley, J. S. (2000). Visual search has memory. Psychological Science, 12, 287-292.

Poisson, M. E., \& Wilkinson, F. (1992). Distractor ratio and grouping processes in visual conjunction search. Perception, 21, 21-38.

Pomplun, M. (1998). Analysis and models of eye movements in comparative visual search. Göttingen: Cuvillier.

Pomplun, M., Reingold, E. M., \& Shen, J. (2001a). Investigating the visual span in comparative search: The effects of task difficulty and divided attention. Cognition, 81, B57-B67.

Pomplun, M., Reingold, E. M., \& Shen, J. (2001b). Peripheral and parafoveal cueing and masking effects on saccadic selectivity in a gaze-contingent window paradigm. Vision Research, 41, 2757-2769.

Pomplun, M., Reingold, E. M., Shen, J., \& Williams, D. E. (2000). The area activation model of saccadic selectivity in visual search. In L. R. Gleitman \& A. K. Joshi (Eds.), Proceedings of the twenty-second annual conference of the cognitive science society (pp. 375-380). Mahwah, NJ: Erlbaum.

Pomplun, M., Velichkovsky, B. M., \& Ritter, H. (1996). Disambiguating complex visual information: Towards communication of personal views of a scene. Perception, 25, 931-948.

Rao, R., Zelinsky, G., Hayhoe, M., \& Ballard, D. (2002). Eye movements in iconic visual search. Vision Research, 42, 1447-1463.

Reingold, E. M., Charness, N., Pomplun, M., \& Stampe, D. M. (2001). Visual span in expert chess players: Evidence from eye movements. Psychological Science, 12, 49-56.

Reingold, E. M., Charness, N., Schultetus, R. S., \& Stampe, D. M. (2001). Perceptual automaticity in expert chess players: Parallel encoding of chess relations. Psychonomic Bulletin and Review, 8, 504-510.

Scialfa, C. T., \& Joffe, K. (1998). Response times and eye movements in feature and conjunction search as a function of eccentricity. Perception \& Psychophysics, 60, 1067-1082.

Shen, J., Reingold, E. M., \& Pomplun, M. (2000). Distractor ratio influences patterns of eye movements during visual search. Perception, 29, 241-250. 
Stampe, D. (1993). Heuristic filtering and reliable calibration methods for video-based pupil tracking systems. Behavior Research Methods, Instruments, \& Computers, 25, 137-142.

Treisman, A. (1982). Perceptual grouping and attention in visual search for features and for objects. Journal of Experimental Psychology: Human Perception \& Performance, 8, 194-214.

Williams, L. G. (1967). The effects of target specification on objects fixated during visual search. Acta Psychologica, 27, 355-360.

Williams, D. E., \& Reingold, E. M. (2001). Preattentive guidance of eye movements during triple-conjunction search tasks. Psychonomic Bulletin and Review, 8, 476-488.

Wolfe, J. M. (1994). Guided search 2.0: A revised model of visual search. Psychonomic Bulletin and Review, 1, 202-238.

Wolfe, J. M. (1996). Extending guided search: Why guided search needs a preattentive item map. In A. F. Kramer, M. G. H. Coles, \& G. D. Logan (Eds.), Converging operations in the study of visual attention (pp. 247-270). Washington, DC: American Psychological Association.

Wolfe, J. M., Cave, K. R., \& Franzel, S. L. (1989). Guided search: An alternative to the feature integration model for visual search. Journal of Experimental Psychology: Human Perception \& Performance, 15, 419-433.

Wolfe, J. M., \& Gancarz, G. (1996). Guided Search 3.0: A model of visual search catches up with Jay Enoch 40 years later. In V. Lakshminarayanan (Ed.), Basic and clinical applications of vision science (pp. 189-192). Dordrecht, The Netherlands: Kluwer Academic Publishers.

Zelinsky, G. J. (1996). Using eye saccades to assess the selectivity of search movements. Vision Research, 36, 2177-2187.

Zelinsky, G., Rao, R., Hayhoe, M., \& Ballard, D. (1997). Eye movements reveal the spatio-temporal dynamics of visual search. Psychological Science, 8, 448-453. 